\title{
Baryon Properties from the CSSM Lattice Collaboration
}

\author{
Waseem Kamleh*, Derek B. Leinweber, M. Selim Mahbub, Benjamin J. Menadue, \\ Thomas Primer, Anthony G. Williams \\ CSSM Lattice Collaboration \\ University of Adelaide, Australia \\ E-mail: waseem.kamleh@adelaide.edu.au
}

\begin{abstract}
We review recent results for baryon properties from lattice QCD arising from the CSSM Lattice Collaboration. Resonance physics is of particular interest. We explore both the positive parity nucleon spectrum, including the $N(1440)$ Roper state, and the negative parity $\Lambda$ spectrum, which contains the low-lying $\Lambda(1405)$. The electromagnetic properties of baryons are also of key interest, and we review the status of our calculations of the neutron magnetic moment obtained by measuring the reponse of the neutron to a uniform background field.
\end{abstract}

Sixth International Conference on Quarks and Nuclear Physics,

April 16-20, 2012

Ecole Polytechnique, Palaiseau, Paris

${ }^{*}$ Speaker. 


\section{Variational Analysis}

The success of the CSSM lattice program in identifying resonance states on the lattice is founded upon variational techniques $[1,2]$ that isolate individual excited states by constructing an operator basis that couples to the states of interest and then considering their cross-correlation matrix in order to diagonalise the operator space. To access $N$ states of the spectrum, we require at least $N$ operators.

The parity-projected, two-point correlation function matrix for $\mathbf{p}=\mathbf{0}$ can be written as

$$
G_{i j}^{ \pm}(t)=\sum_{\mathbf{x}} \operatorname{tr}_{\mathrm{sp}}\left(\Gamma_{ \pm}\left\langle\Omega\left|\chi_{i}(x) \bar{\chi}_{j}(0)\right| \Omega\right\rangle\right)=\sum_{\alpha=0}^{N-1} \lambda_{i}^{\alpha} \bar{\lambda}_{j}^{\alpha} \mathrm{e}^{-m_{\alpha} t}
$$

where $\Gamma_{ \pm}$are the parity-projection operators and $\lambda_{i}^{\alpha}$ and $\bar{\lambda}_{j}^{\alpha}$ are, respectively, the couplings of interpolators $\chi_{i}$ and $\bar{\chi}_{j}$ at the sink and source to eigenstates $\alpha=0, \ldots, N-1$ of mass $m_{\alpha}$. The idea now is to construct $N$ independent operators $\phi_{i}$ that isolate $N$ baryon states $\left|B_{\alpha}\right\rangle$; that is, to find operators $\bar{\phi}^{\alpha}=\sum_{i=1}^{N} u_{i}^{\alpha} \bar{\chi}_{i}$ and $\phi^{\alpha}=\sum_{i=1}^{N} v_{i}^{\alpha *} \chi_{i}$ such that

$$
\begin{aligned}
\left\langle B_{\beta}, p, s\left|\bar{\phi}^{\alpha}\right| \Omega\right\rangle & =\delta_{\alpha \beta} \bar{z}^{\alpha} \bar{u}(\alpha, p, s), \text { and } \\
\left\langle\Omega\left|\phi^{\alpha}\right| B_{\beta}, p, s\right\rangle & =\delta_{\alpha \beta} z^{\alpha} u(\alpha, p, s),
\end{aligned}
$$

where $z^{\alpha}$ and $\bar{z}^{\alpha}$ are the coupling strengths of $\phi^{\alpha}$ and $\bar{\phi}^{\alpha}$ to the state $\left|B_{\alpha}\right\rangle$. It follows that

$$
G_{i j}^{ \pm}(t) u_{j}^{\alpha}=\lambda_{i}^{\alpha} \bar{z}^{\alpha} \mathrm{e}^{-m_{\alpha} t},
$$

where, for notational convenience, we take the repeated Latin indices to be summed over while repeated Greek indices are not.

The only $t$ dependence in Eq. (1.3) is in the exponential term, so we immediately construct the recurrence relation $G_{i j}^{ \pm}(t) u_{j}^{\alpha}=\mathrm{e}^{-m_{\alpha} \Delta t} G_{i k}^{ \pm}(t+\Delta t) u_{k}^{\alpha}$, which can be written as

$$
\left(G^{ \pm}(t+\Delta t)\right)^{-1} G^{ \pm}(t) \mathbf{u}^{\alpha}=\mathrm{e}^{-m_{\alpha} \Delta t} \mathbf{u}^{\alpha} .
$$

This is an eigensystem equation for the matrix $\left(G^{ \pm}(t+\Delta t)\right)^{-1} G^{ \pm}(t)$, with eigenvectors $\mathbf{u}^{\alpha}$ and eigenvalues $\mathrm{e}^{-m_{\alpha} \Delta t}$.

Similarly, we can construct the associated left-eigensystem equation $\mathbf{v}^{\alpha \dagger} G^{ \pm}(t)\left(G^{ \pm}(t+\Delta t)\right)^{-1}=$ $\mathrm{e}^{-m_{\alpha} \Delta t} \mathbf{v}^{\alpha \dagger}$, and then Eq. (1.2) implies that

$$
G_{\alpha}^{ \pm}(t):=\mathbf{v}^{\alpha \dagger} G^{ \pm}(t) \mathbf{u}^{\alpha}=z^{\alpha} \bar{z}^{\alpha} \mathrm{e}^{-m_{\alpha} t} .
$$

Thus, the only state present in $G_{\alpha}^{ \pm}(t)$ is $\left|B_{\alpha}\right\rangle$ of mass $m_{\alpha}$.

\section{Nucleon Spectrum}

The first positive-parity excited state of the nucleon, known as the Roper resonance, $N \frac{1}{2}^{+}(1440$ $\mathrm{MeV}$ ) $\mathrm{P}_{11}$, has presented a long-standing puzzle since its discovery in the 1960's due to its lower mass compared to the adjacent negative parity, $N \frac{1}{2}^{-}(1535 \mathrm{MeV}) \mathrm{S}_{11}$, state. In constituent quark models with harmonic oscillator potentials, the lowest-lying odd-parity state naturally occurs below the $\mathrm{P}_{11}$ state [3, 4]. In nature the Roper resonance is almost $100 \mathrm{MeV}$ below the $\mathrm{S}_{11}$ state. 

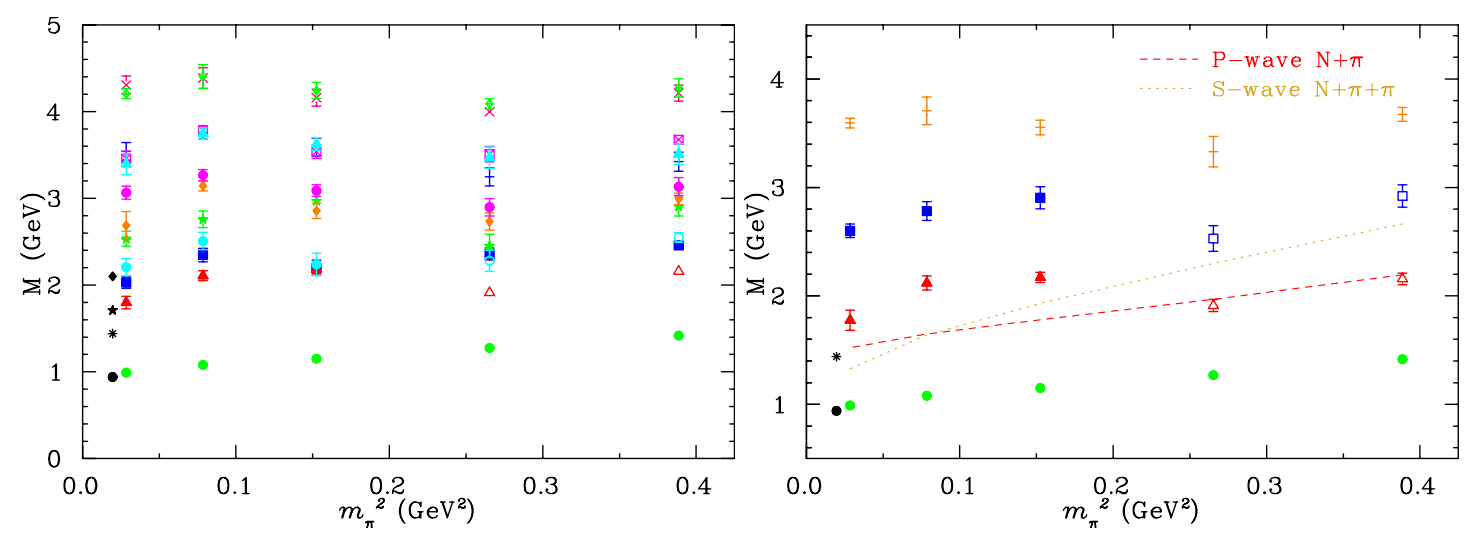

Figure 1: The even parity nucleon spectrum in full QCD. (Left) The mass spectrum resulting from the superposition of two $8 \times 8$ correlation matrix analyses. (Right) The low-lying nucleon spectrum resulting from a $4 \times 4$ correlation matrix analysis. Scattering channels are shown to demonstrate that the first excited state on the lattice is consistent with the Roper resonance.

Using the variational techniques described above, the CSSM [5, 6, 7] has successfully isolated the Roper on the lattice, first in quenched QCD[8], then in full QCD[9]. The highlights of the CSSM results for the even-parity nucleon spectrum in Full QCD are shown in Figure 1. These results (and the full QCD results that follow below) were calculated on the $2+1$ flavour nonperturbatively improved clover configurations[10] made available by the PACS-CS collaboration via the ILDG[11]. Critical to our results is the construction of a large operator basis by considering different amounts of gauge-invariant Gaussian smearing[12].

\section{Lambda Spectrum}

The $1405 \mathrm{MeV}$ resonance of the Lambda baryon has puzzled researchers for many years. It is the lowest-lying excited state of the Lambda, and yet it has negative parity (a property associated with angular momentum). Moreover, it lies lower than the lowest negative-parity state of the nucleon, even though it has valence strange quarks. It also lacks a nearby spin-orbit partner, with the lowest spin-3/2 $2^{-}$state being the $\Lambda(1520)$. The internal structure of this resonance has remained a mystery for many years. On the one hand, it is regarded as a conventional three-quark state, while on the other it is interpreted as a kaon-nucleon bound state. There have so far been several Lattice QCD studies of this resonance $[13,14,15,16]$, however most of these have used the quenched approximation, and very few have managed to identity the mass-suppression associated with the $\Lambda(1405)$.

The highlights of the CSSM results for the odd-parity $\Lambda$ spectrum[17] calculated in full QCD on the PACS-CS configurations are shown in Figure 2. The results in the left plot use the isospin-0 "common" interpolating fields $\chi_{1}^{\mathrm{c}}$ and $\chi_{2}^{\mathrm{c}}$ [18] that make no assumptions about SU(3)-flavour symmetry, whereas the results in the right plot use the traditional purely octet $\left(\chi_{1}^{8}\right.$ and $\left.\chi_{2}^{8}\right)$ and purely singlet $\left(\chi^{1}\right)$ operators to isolate all three low-lying states. Note that on the PACS-CS configurations the kaon mass comes out a little too high. We correct for this in our $\Lambda$ results by adjusting the $\kappa_{s}$ value for the strange quark propagators in order to correctly reproduce the kaon mass. 

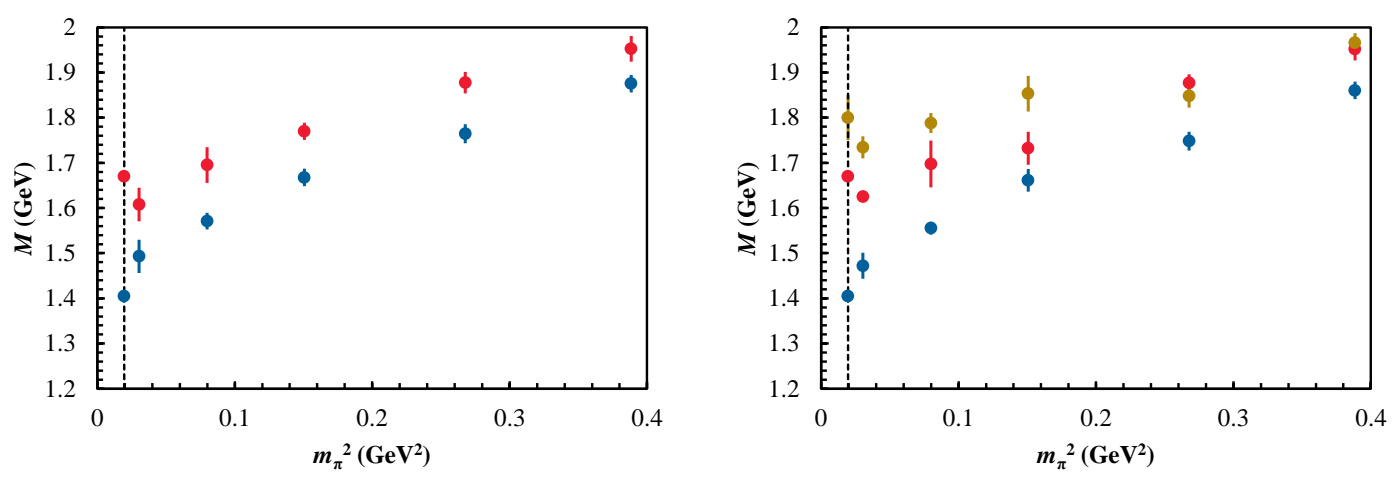

Figure 2: Results for the low-lying odd-parity $\Lambda$ spectrum (with corrected $s$-quark mass), making use of three different smearing values. (Left) Results from the $6 \times 6$ correlation matrix analysis using $\chi_{1}^{c}$ and $\chi_{2}^{c}$. (Right) Results from the $9 \times 9$ correlation matrix analysis using $\chi_{1}^{8}, \chi_{2}^{8}$ and $\chi^{1}$.

\section{Neutron Magnetic Moment}

The magnetic moment and magnetic polarisability are fundamental properties of a particle that describe its response to an applied magnetic field. The background field method is a well-known technique for examining these properties via lattice QCD and elsewhere[19, 20, 21, 22, 23, 24]. In this method a uniform background magnetic field is imposed on the lattice in the form of a $U(1)$ phase factor applied to the usual QCD gauge field. This causes a shift in the calculated ground-state energy of the particle which is combined with an energy relation to extract the magnetic moment reported here.

The CSSM results for the energy difference between the spin-up and spin-down neutron calculated on the four heaviest PACS-CS ensembles are shown in Figure 3. For each quark mass, we perform a fit of the energy-difference as a function of background field strength, using a linear plus cubic fit function. The magnetic moment is derived from the coefficient of the linear term in the fits. The full QCD results for the neutron magnetic moment as a function of $m_{\pi}^{2}$ are shown in Figure 4. Results from a previous quenched study[25] using the background field method are shown for comparison. It is anticipated that there are significant finite-volume corrections that need to be made in order to reach the physical value.

\section{Acknowledgements}

This research was undertaken on the NCI National Facility in Canberra, Australia, which is supported by the Australian Commonwealth Government. We also acknowledge eResearch SA for grants of supercomputing time. This research is supported by the Australian Research Council.

\section{References}

[1] C. Michael, Nucl. Phys. B 259, 58 (1985)

[2] M. Lüscher and U. Wolff, Nucl. Phys. B 339, 222 (1990)

[3] N. Isgur and G. Karl, Phys.Lett. B72, 109 (1977) 

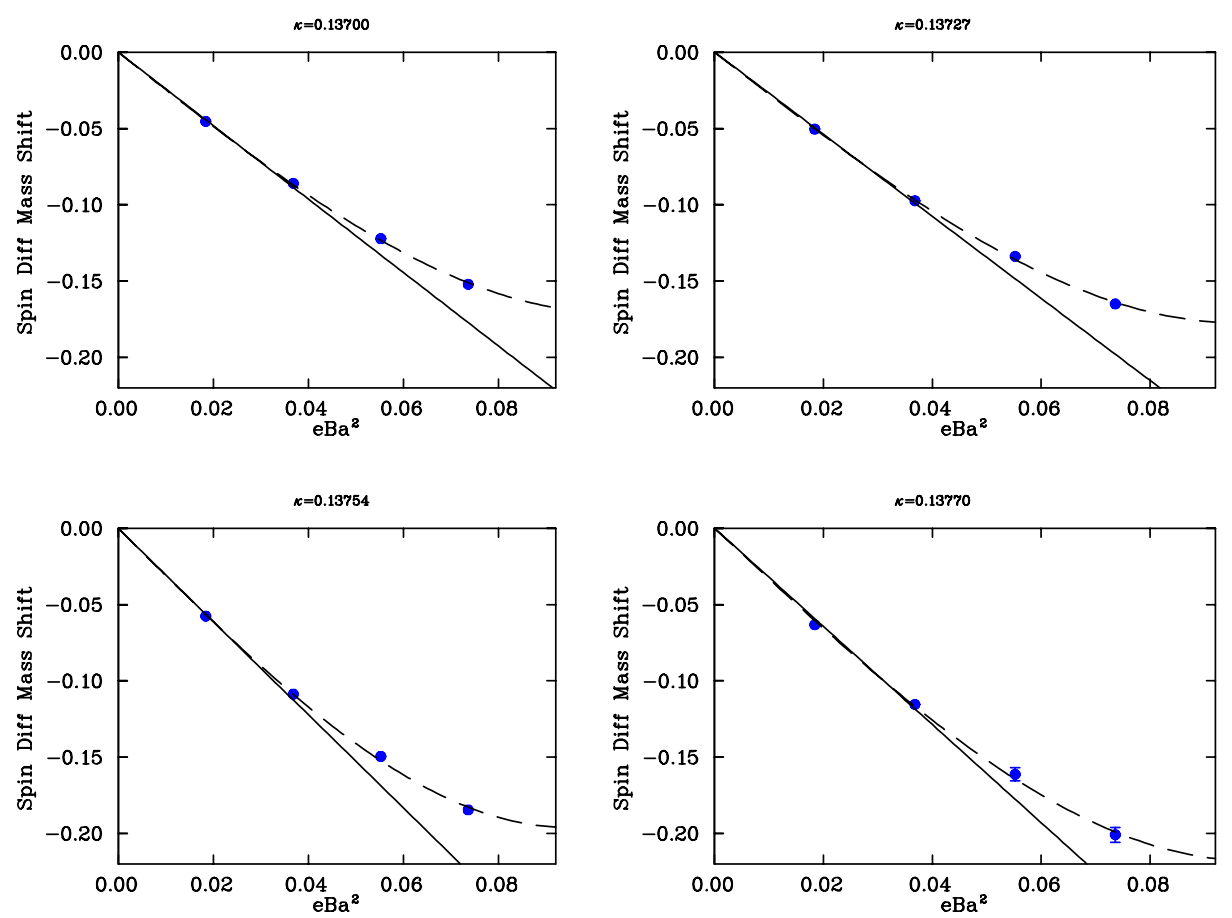

Figure 3: The energy difference between the spin-up and spin-down neutron as a function of background field strength, with linear plus cubic fits. Results are shown for the four heaviest PACS-CS masses.

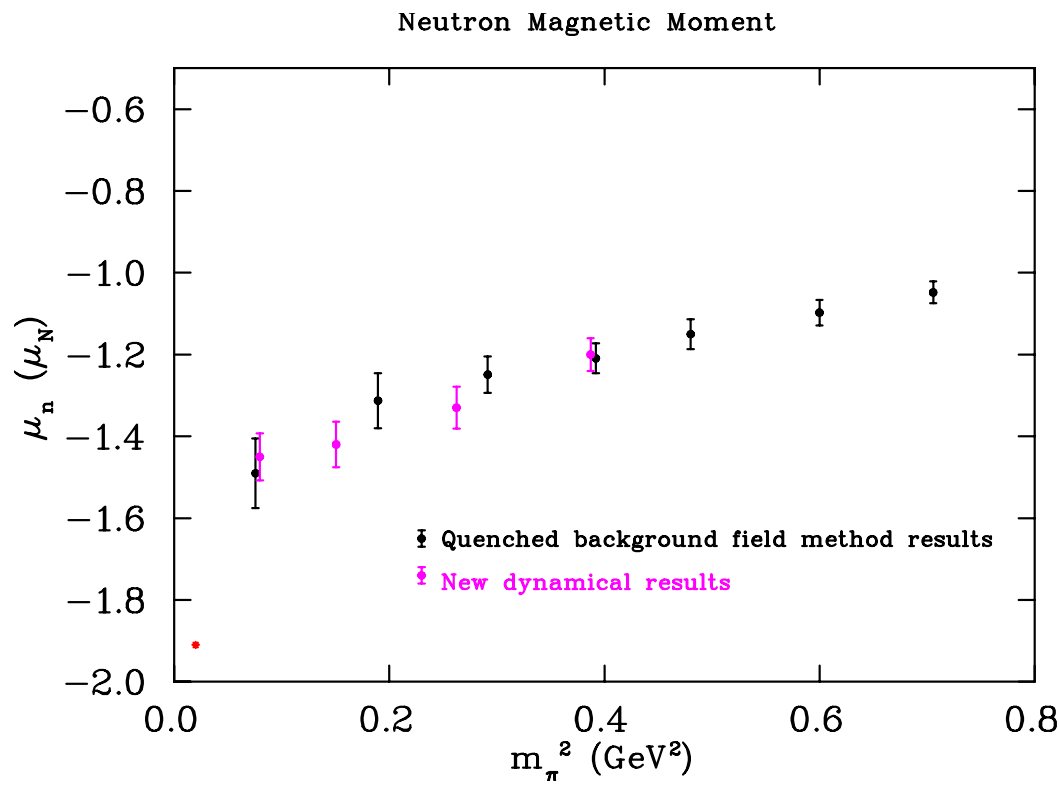

Figure 4: Results for the neutron magnetic moment as a function of $m_{\pi}^{2}$, using the background field method. Current full QCD results are compared against older quenched results using the same method. 
[4] N. Isgur and G. Karl, Phys.Rev. D19, 2653 (1979)

[5] M. Mahbub, A. O. Cais, W. Kamleh, B. Lasscock, D. B. Leinweber, et al., Phys.Rev. D80, 054507 (2009), arXiv:0905.3616 [hep-lat]

[6] M. Mahbub, A. O. Cais, W. Kamleh, D. B. Leinweber, and A. G. Williams, Phys.Rev. D82, 094504 (2010), arXiv:1004.5455 [hep-lat]

[7] M. Mahbub, W. Kamleh, D. B. Leinweber, A. O Cais, and A. G. Williams, Phys.Lett. B693, 351 (2010), arXiv:1007.4871 [hep-lat]

[8] M. Mahbub, A. O. Cais, W. Kamleh, B. G. Lasscock, D. B. Leinweber, et al., Phys.Lett. B679, 418 (2009), arXiv:0906.5433 [hep-lat]

[9] M. S. Mahbub, W. Kamleh, D. B. Leinweber, P. J. Moran, and A. G. Williams (CSSM Lattice collaboration), Phys.Lett. B707, 389 (2012), arXiv:1011.5724 [hep-lat]

[10] S. Aoki, K.-I. Ishikawa, N. Ishizuka, T. Izubuchi, D. Kadoh, K. Kanaya, Y. Kuramashi, Y. Namekawa, M. Okawa, Y. Taniguchi, A. Ukawa, N. Ukita, and T. Yoshié (PACS-CS Collaboration), Phys. Rev. D 79, 034503 (Feb 2009), arXiv:0807.1661 [hep-lat]

[11] M. G. Beckett, B. Joo, C. M. Maynard, D. Pleiter, O. Tatebe, et al., Comput.Phys.Commun. 182, 1208 (2011), arXiv:0910.1692 [hep-lat]

[12] S. Güsken, Nucl. Phys. Proc. Suppl. 17, 361 (1990)

[13] W. Melnitchouk, S. Bilson-Thompson, F. D. R. Bonnet, J. N. Hedditch, F. X. Lee, D. B. Leinweber, A. G. Williams, J. M. Zanotti, and J. B. Zhang, Phys. Rev. D 67, 114506 (Jun 2003), arXiv:hep-lat/0202022

[14] Y. Nemoto, N. Nakajima, H. Matsufuru, and H. Suganuma, Phys. Rev. D 68, 094505 (Nov 2003), arXiv:hep-lat/0302013

[15] T. Burch, C. Gattringer, L. Y. Glozman, C. Hagen, D. Hierl, C. B. Lang, and A. Schäfer (BGR [Bern-Graz-Regensburg] Collaboration), Phys. Rev. D 74, 014504 (Jul 2006), arXiv:hep-lat/0604019

[16] N. Ishii, T. Doi, M. Oka, and H. Suganuma, Prog. Theor. Phys. Suppl. 168, 598 (2007), arXiv:0707.0079 [hep-lat]

[17] B. J. Menadue, W. Kamleh, D. B. Leinweber, and M. S. Mahbub, Phys.Rev.Lett. 108, 112001 (2012), arXiv:1109.6716 [hep-lat]

[18] D. B. Leinweber, R. Woloshyn, and T. Draper, Phys.Rev. D43, 1659 (1991)

[19] C. W. Bernard, T. Draper, K. Olynyk, and M. Rushton, Phys.Rev.Lett. 49, 1076 (1982)

[20] G. Martinelli, G. Parisi, R. Petronzio, and F. Rapuano, Phys.Lett. B116, 434 (1982)

[21] H. Rubinstein, S. Solomon, and T. Wittlich, Nucl.Phys. B457, 577 (1995), arXiv:hep-lat/9501001 [hep-lat]

[22] F. Lee, R. Kelly, L. Zhou, and W. Wilcox, Phys.Lett. B627, 71 (2005), arXiv:hep-lat/0509067 [hep-lat]

[23] F. X. Lee, L. Zhou, W. Wilcox, and J. C. Christensen, Phys.Rev. D73, 034503 (2006), arXiv:hep-lat/0509065 [hep-lat]

[24] M. Burkardt, D. B. Leinweber, and X.-m. Jin, Phys.Lett. B385, 52 (1996), arXiv:hep-ph/9604450 [hep-ph]

[25] T. Primer, W. Kamleh, and D. B. Leinweber, AIP Conf.Proc. 1354, 216 (2011) 